\title{
Diferenças no consumo alimentar nas áreas urbanas e rurais do Brasil: Pesquisa Nacional de Saúde
}

\author{
Food consumption differences in Brazilian urban and rural areas: \\ the National Health Survey
}

Danielle Vasconcellos de Paula Costa (https://orcid.org/0000-0003-0370-4612) ${ }^{1}$

Mariana Souza Lopes (https://orcid.org/0000-0003-3128-7959) ${ }^{2}$

Raquel de Deus Mendonça (https://orcid.org/0000-0001-7599-8715) ${ }^{3}$

Deborah Carvalho Malta (https://orcid.org/0000-0002-8214-5734) ${ }^{4}$

Patrícia Pinheiro de Freitas (https://orcid.org/0000-0001-9355-3066) ${ }^{5}$

Aline Cristine Souza Lopes (https://orcid.org/0000-0001-9782-2606) ${ }^{2}$

${ }^{1}$ Escola de Enfermagem, Universidade Federal de Minas Gerais (UFMG). Av. Alfredo Balena 190, Santa Efigênia. 30130-100 Belo Horizonte MG Brasil. daniellenutri@gmail.com ${ }^{2}$ Grupo de Pesquisa de Intervenções em Nutrição, Departamento de Nutrição, Escola de Enfermagem, UFMG. Belo Horizonte MG Brasil.

${ }^{3}$ Faculdades Kennedy. Belo Horizonte MG Brasil. ${ }^{4}$ Departamento Materno Infantil e Saúde Pública, Escola de Enfermagem, UFMG. Belo Horizonte MG Brasil.

${ }^{5}$ Grupo de Pesquisa de Intervenções em

Nutrição, Programa de

Pós-Graduação em Saúde

Pública, Faculdade de

Medicina, UFMG. Belo

Horizonte MG Brasil.
Abstract This paper aimed to identify food consumption differences as per healthy and unhealthy diet markers among adults living in Brazilian urban and rural areas. A cross-sectional study was performed with data from the National Health Survey (2013). Diet was assessed by using healthy and unhealthy diet markers. Prevalence (\%) was estimated, and sequential logistic regression models were adjusted to estimate odds ratios (OR) and confidence intervals $(95 \% \mathrm{CI})$. Urban areas evidenced a higher consumption of fruits and vegetables, fish, soft drinks, and meal replacement by snacks, while rural areas showed higher consumption of meat with excess fat and beans. Adjusted analyses showed higher regular consumption of beans and meat with excess fat; and lower consumption of soft drinks, fruits and vegetables and meal replacement by snacks in rural areas compared to urban areas. Similar trends were observed in the macro-regions of the country. Food consumption differences among Brazilians living in rural and urban areas denote the importance of fostering food policies that respect and value food traditions and culture.

Key words Food Consumption, Nutrition Surveys, Rural Area, Urban Area, Health status disparities
Resumo Objetivou-se identificar diferenças no consumo alimentar, segundo marcadores de alimentação saudável e não saudável, entre adultos residentes nas áreas urbanas e rurais do Brasil. Realizou-se estudo transversal com dados da Pesquisa Nacional de Saúde (2013). A alimentação foi avaliada por marcadores de alimentação saudável e não saudável. Estimou-se as prevalências (\%) e modelos de regressão logística sequencial foram ajustados para estimar odds ratio $(O R) e$ intervalos de confiança (IC95\%). Verificou-se maior consumo de frutas e hortaliças, peixes, refrigerantes e substituições de refeições nas áreas urbanas, enquanto que, o consumo de carne com gordurase feijão foi maior nas áreas rurais. Análises ajustadas mostraram maior consumo regular de feijão e de carne com excesso de gordura; e menor consumo de refrigerantes, de frutas e hortaliças e de substituição de refeições por lanches nas áreas rurais em comparação com as urbanas. Tendências semelhantes foram observadas nas macrorregiões do país. As diferenças no consumo alimentar de brasileiros residentes em áreas rurais e urbanas denotam a importância de fomentar políticas de alimentação que respeitem e valorizem as tradições e a cultura alimentar.

Palavras-chave Consumo de Alimentos, Inquéritos Nutricionais, Zona Rural, Área Urbana, Disparidades nos niveis de saúde 


\section{Introdução}

As doenças crônicas não transmissíveis (DCNT) constituem crescente problema mundial de saúde. Um dos fatores que mais contribuem para este aumento progressivo são os estilos de vida pouco saudáveis, principalmente a alimentação inadequada ${ }^{1}$.

Apesar da Organização Mundial da Saúde (OMS) alertar há mais de quinze anos sobre a necessidade de promover melhorias na alimentação das populações ${ }^{2}$, o padrão alimentar mundial se deteriora progressivamente, sobretudo nas áreas urbanas $^{3}$. As mudanças no consumo alimentar parecem advir do ritmo de vida acelerado e intensas transformações no sistema alimentar, que promovem ascensão do consumo de alimentos ultraprocessados ${ }^{4}$ em detrimento dos in natura e minimamente processados ${ }^{5-8}$.

Mais da metade das pessoas do mundo vive em áreas urbanas ${ }^{9}$, no Brasil chega a $80 \%{ }^{10}$. O Brasil é um país de dimensões continentais, com variações regionais significativas e um patrimônio culinário expresso em hábitos e receitas tradicionais ${ }^{11}$. Porém, os processos de urbanização e industrialização desiguais parecem afetar de forma diferenciada os grupos populacionais ${ }^{11,12}$ e, provavelmente, as macrorregiões do país. De tal modo que, avaliar as diferenças no consumo alimentar dos brasileiros que vivem em áreas urbanas e rurais é importante, sobretudo pelo país apresentar contínuo e sistemático esforço para garantir o Direito Humano a Alimentação Adequada e Saudável e a soberania alimentar mediante a valorização e respeito à cultura alimentar.

Entretanto, são escassas as evidências sobre as distinções alimentares nas áreas urbanas e rurais, segundo as macrorregiões brasileiras. Neste sentido, este artigo objetivou identificar diferenças no consumo alimentar, segundo marcadores de alimentação saudável e não saudável, entre adultos residentes nas áreas urbanas e rurais do Brasil.

\section{Métodos}

\section{Delineamento e população de estudo}

Realizou estudo transversal com dados da Pesquisa Nacional de Saúde ${ }^{13}$, conduzida pelo Instituto Brasileiro de Geografia e Estatística (IBGE) em parceria com o Ministério da Saúde, e parte do Sistema Integrado de Pesquisas Domiciliares (SIPD). A PNS se destaca por ser conduzida face a face em amostra representativa da população brasileira e de suas macrorregiões, nas áreas urbanas e rurais, configurando-se como o maior inquérito nacional sobre saúde. A PNS foi aprovada pela Comissão Nacional de Ética em Pesquisa para Seres Humanos, do Ministério da Saúde, e o Termo de Consentimento Livre e Esclarecido foi assinado por todos os entrevistados.

A PNS utiliza processo de amostragem aleatória simples, por conglomerados em três estágios: (1) unidades primárias de amostragem (UPA) compostas por um ou mais setores censitários; (2) domicílios presentes em cada UPA selecionada no primeiro estágio; (3) morador adulto $(\geq 18$ anos) selecionado em cada domicílio 5 .

A coleta de dados ocorreu entre agosto de 2013 e fevereiro de 2014 em Personal Digital Assistance (PDA). Foram realizadas 64.348 entrevistas domiciliares e 60.202 entrevistas individuais com o morador adulto selecionado ${ }^{13}$. Maior detalhamento metodológico pode ser obtido em Souza-Júnior et al. ${ }^{5}$.

\section{Variáveis desfechos: marcadores de alimentação saudável e não saudável}

O consumo alimentar foi analisado por três marcadores de alimentação saudável e quatro, não saudável. Os marcadores de alimentação saudável foram: consumo recomendado de frutas e hortaliças (cinco ou mais vezes ao dia em cinco ou mais dias da semana); consumo regular de feijão (cinco dias ou mais por semana) e de peixe (pelo menos uma vez por semana). Já os marcadores de alimentação não saudável foram: ingestão de carne com gordura ou frango com pele (sim); consumo regular (cinco dias ou mais por semana) de refrigerantes ou suco artificial, consumo regular de alimentos doces; substituição de refeições por lanches (substituição do almoço ou jantar por sanduíches, salgados ou pizzas, sete dias por semana).

A classificação do consumo em recomendado e regular foi baseada no sistema de Vigilância de Fatores de Risco e Proteção para Doenças Crônicas por Inquérito Telefônico (VIGITEL). E estes indicadores tiveram sua validade analisada ${ }^{14}$.

\section{Variável explicativa principal: situação do domicílio}

A situação do domicílio na PNS é definida pela sua localização em áreas urbanas ou rurais de acordo com a lei municipal vigente à época da realização do Censo Demográfico. A situação 
urbana abrange as áreas correspondentes às cidades (sedes municipais), vilas (sedes distritais) ou às áreas urbanas isoladas; enquanto que, a situação rural abrange toda a área situada fora desses limites ${ }^{10}$.

\section{Covariáveis}

Foram utilizadas como variáveis de ajustamento os seguintes dados sociodemográficos: sexo (masculino e feminino), faixa etária (18-29, 30-39, 40-49, 50-59 e 60 ou mais), nível de instrução (sem e com pouca instrução: sem instrução e ensino fundamental incompleto, instrução básica completa: ensino fundamental completo e médio incompleto, instrução superior incompleta: ensino médio completo e superior incompleto, instrução superior completa: ensino superior completo), cor da pele (preto, pardo e branco) e macrorregiões do país (Norte, Nordeste, Sul, Sudeste e Centro-Oeste).

\section{Análise dos dados}

As análises foram realizadas no software Stata (Stata Corporation, College Station, Texas) versão 14.0 utilizando o comando $s v y$, que considera o delineamento complexo da amostra. Realizou-se inicialmente análise descritiva das variáveis pelo cálculo de prevalência e intervalo de confiança a 95\%.

Para verificar a associação entre local de residência e marcadores de alimentação saudável e não saudável foram construídos modelos ajustados de regressão logística sequencial. O Modelo 1 foi ajustado pelas variáveis sexo e idade; Modelo 2 pelas variáveis do Modelo 1 adicionado de escolaridade e cor da pele; e o Modelo 3 ajustado pelas variáveis do Modelo 2 adicionado das macrorregiões do país. Os resultados foram apresentados pelos valores de odds ratio e intervalo de confiança a 95\%.

\section{Resultados}

A população brasileira reside majoritariamente nas áreas urbanas (81,0\%; IC95\%: 80,8-81,2). A proporção de indivíduos residentes nas áreas rurais do país foi superior apenas nas macrorregiões Norte e Nordeste (Tabela 1).

Nas áreas rurais verificou-se predominância de indivíduos do sexo masculino (51,4\%, IC95\%: 49,7-53,0 vs. 46,5\%, IC95\%: 45,6-47,3), com cor de pele parda (54,6\%, IC95\%: 52,6-56,5 vs.
40,6\%, IC95\%: 39,8-41,5), sem ou com pouca instrução (66,0\%, IC95\%: 64,1-67,7 vs. 34,7\%, IC95\%: 33,8-35,6) quando comparada às áreas urbanas (Tabela 1).

Ao analisar as prevalências dos marcadores do consumo alimentar saudável e não saudável no Brasil, observou-se nas áreas rurais, em comparação com as urbanas, menor consumo de frutas e hortaliças, peixes, refrigerantes e substituição das refeições por lanches; e maior consumo de feijão, e carne ou frango com excesso de gordura, sem diferenças para o consumo de alimentos doces (Tabela 2). Observou-se ainda, diferenças entre as macrorregiões, sendo o consumo de peixe maior nas regiões Norte e Nordeste, e o de feijão nas regiões Sudeste e Centro-Oeste. Já na macrorregião Nordeste verificou-se menor consumo de frutas e hortaliças.

Ao analisar estes marcadores segundo as macrorregiões do país foram observadas além de variações das prevalências, diferenças na magnitude. Por exemplo, no Norte do país foi maior a prevalência de consumo regular de peixes nas áreas rurais (78,9\%, IC95\%: 77,3-80,3 vs. 73,8\%, IC95\%: 72,9-74,7); e o consumo de doces nas áreas urbanas das macrorregiões Norte $(12,2 \%$, IC95\%: 11,6-12,9 vs. 9,0\%, IC95\%: 8,0-10,1) e Nordeste (18,3\%, IC95\%:17,7-19,0 vs. 15,0\%, IC95\%:14,0-16,1) quando comparadas às áreas rurais (Tabela 2).

$\mathrm{Na}$ análise ajustada dos marcadores de consumo alimentar saudável e não saudável, observou-se nas áreas rurais um maior consumo de feijão (OR=1,20; IC95\%: 1,14-1,26) e de carne ou frango com excesso de gordura $(\mathrm{OR}=1,48$; IC95\%: 1,42-1,55); e menor de frutas e hortaliças $(\mathrm{OR}=0,89$; IC95\%: 0,85-0,96); peixes $(\mathrm{OR}=0,88$; IC95\%: 0,84-0,92); refrigerantes $(\mathrm{OR}=0,55$; IC95\%: 0,52-0,59) e de substituição de refeições por lanches (OR=0,59; IC95\%: 0,51-0,66) (Tabela 3).

\section{Discussão}

Há diferenças no consumo alimentar, segundo marcadores de alimentação saudável e não saudável, entre adultos residentes nas áreas urbanas e rurais do Brasil, e macrorregiões. Brasileiros residentes nas áreas rurais têm maior chance de manter um padrão alimentar tradicional, com o consumo de alimentos minimamente processados, especialmente feijão; e menor consumo de alimentos ultraprocessados; apesar do menor consumo de frutas e hortaliças, e de peixes. 
Tabela 1. Descrição da amostra de acordo com características sociodemográficas e região. Pesquisa Nacional de Saúde, Brasil, 2013 (N=60.202).

\begin{tabular}{|c|c|c|c|c|c|c|}
\hline \multirow{2}{*}{ Variável } & \multicolumn{2}{|c|}{ Brasil } & \multicolumn{2}{|c|}{ Urbano } & \multicolumn{2}{|c|}{ Rural } \\
\hline & $\%$ & IC95\% & $\%$ & IC95\% & $\%$ & IC95\% \\
\hline \multicolumn{7}{|l|}{ Sexo } \\
\hline Masculino & 47,2 & $46,4-47,9$ & 46,5 & $45,6-47,3$ & 51,4 & $49,7-53,0$ \\
\hline Feminino & 52,8 & $52,1-53.6$ & 53,5 & $52,6-53,3$ & 48,6 & $48,6-50,3$ \\
\hline \multicolumn{7}{|l|}{ Idade (anos) } \\
\hline 18-29 & 26,1 & $25,4-26,7$ & 26,2 & $25,6-26,9$ & 25,2 & $23,7-26,7$ \\
\hline $30-39$ & 21,6 & $21,0-22,2$ & 21,6 & $20,9-22,2$ & 21,8 & $20,6-23,0$ \\
\hline $40-49$ & 18,1 & $17,5-18,6$ & 18,0 & $17,4-18,3$ & 18,4 & $17,3-19,5$ \\
\hline $50-59$ & 16,2 & $15,6-16,7$ & 16,4 & $15,7-17,0$ & 15,2 & $14,2-16,4$ \\
\hline$\geq 60$ & 18,0 & $17,4-18,6$ & 17,8 & $17,1-18,5$ & 19,4 & $19,3-20,5$ \\
\hline \multicolumn{7}{|l|}{ Cor da pele } \\
\hline Branca & 48,1 & $47,3-48,9$ & 50,1 & $49,2-50,9$ & 36,1 & $34,2-38,0$ \\
\hline Pardo & 42,6 & $41,8-43,3$ & 40,6 & $39,8-41,5$ & 54,6 & $52,6-56,5$ \\
\hline Preto & 9,3 & $8,9-9,8$ & 9,3 & $8,8-9,8$ & 9,3 & $8,0-10,7$ \\
\hline \multicolumn{7}{|l|}{ Escolaridade } \\
\hline Sem e com pouca instrução ${ }^{1}$ & 39,0 & $38,2-39,9$ & 34,7 & $33,8-35,6$ & 66,0 & $64,1-67,7$ \\
\hline Instrução básica completa² & 15,6 & $15,0-16,1$ & 15,7 & $15,1-16,3$ & 14,8 & $13,5-16,2$ \\
\hline Instrução superior incompleta ${ }^{3}$ & 32,7 & $32,0-33,5$ & 35,4 & $34,5-36,2$ & 16,3 & $15,0-17,7$ \\
\hline Instrução superior completa ${ }^{4}$ & 12,7 & $12,0-13,4$ & 14,2 & $13,4-15,1$ & 2,9 & $2,3-3,4$ \\
\hline \multicolumn{7}{|l|}{ Região } \\
\hline Norte & 7,4 & $7,2-8,6$ & 6,7 & $6,5-6,9$ & 11,7 & $10,9-12,5$ \\
\hline Nordeste & 26,6 & $26,1-27,1$ & 23,5 & $23,0-24,0$ & 45,8 & $44,0-17,6$ \\
\hline Sudeste & 43,8 & $43,1-44,4$ & 47,3 & $46,6-48,0$ & 21,8 & $50,1-23,6$ \\
\hline Sul & 14,9 & $14,3-15,2$ & 14,7 & $14,2-15,1$ & 16,0 & $14,8-17,2$ \\
\hline Centro-Oeste & 7,3 & $7,1-7,5$ & 7,8 & $7,5-8,0$ & 4,7 & $4,3-5,1$ \\
\hline
\end{tabular}

${ }^{1}$ Sem e com pouca instrução (sem instrução e ensino fundamental incompleto); ${ }^{2}$ Instrução básica completa (Ensino fundamental completo e médio incompleto); ${ }^{3}$ Instrução superior incompleta (Ensino médio completo e superior incompleto); ${ }^{4}$ Instrução superior completa (Ensino superior completo).

Fonte: Elaborado pelas autoras, 2019.

Estas diferenças no padrão de consumo alimentar verificadas entre as áreas urbanas e rurais também foram verificadas nas macrorregiões do país. Destaca-se apenas a macrorregião Norte, que o maior consumo de peixes foi verificado nas áreas rurais e não urbanas, conforme observado no restante do país e demais macrorregiões.

O Brasil possui grande extensão territorial e diferenças de clima, cultura e atividades econômicas em suas macrorregiões, produzindo marcas nacionais e regionais, as quais provavelmente refletem na diversidade de hábitos alimentares da população brasileira. Como marcas nacionais identificadas neste estudo citam-se o consumo de feijão, e como marcas regionais, o consumo de peixes.

Outro estudo conduzido com dados da PNS, mas com o objetivo de analisar a prevalência de consumo de alimentos considerados marcadores saudáveis na população brasileira como um todo revelou que ainda se mantém no país hábitos alimentares saudáveis. Verificou-se que, aproximadamente três quartos da população consumiam feijão regularmente, pouco mais de um terço apresentavam consumo de frutas e hortaliças recomendado e o consumo regular de peixe foi referido por um pouco mais da metade da população ${ }^{15}$.

Neste estudo foi observado maior consumo de feijão nas áreas rurais de todas as macrorregiões do país, exceto na Norte, que o consumo não diferiu entre as áreas. O feijão é um importante marcador da cultura alimentar de toda população brasileira e de alimentação saudável ${ }^{16-18}$, um símbolo da comida básica e cotidiana dos brasileiros. Estes resultados podem sugerir que o processo de urbanização e as mudanças do padrão alimentar na contemporaneidade podem, de certa forma, contribuir para a redução do consumo desse alimento minimamente processado, cujo 
Tabela 2. Prevalência de marcadores da alimentação saudável e não saudável por região do país e situação do domicílio. Pesquisa Nacional de Saúde, Brasil, 2013.

\begin{tabular}{|c|c|c|c|c|c|c|}
\hline \multirow{3}{*}{ Marcadores da alimentação } & \multicolumn{6}{|c|}{ Prevalência \% (IC95\%) } \\
\hline & \multicolumn{2}{|c|}{ Brasil } & \multicolumn{2}{|c|}{ Norte } & \multicolumn{2}{|c|}{ Nordeste } \\
\hline & Urbano & Rural & Urbano & Rural & Urbano & Rural \\
\hline \multicolumn{7}{|l|}{ Saudáveis } \\
\hline $\begin{array}{l}\text { Consumo recomendado de } \\
\text { FH }\end{array}$ & $\begin{array}{c}38,2 \\
(37,3-39,2)\end{array}$ & $\begin{array}{c}31,2 \\
(29,4-33,0)\end{array}$ & $\begin{array}{c}37,1 \\
(36,1-38,0)\end{array}$ & $\begin{array}{c}30,4 \\
(28,8-32,1)\end{array}$ & $\begin{array}{c}29,1 \\
(28,4-30,0)\end{array}$ & $\begin{array}{c}22,6 \\
(21,4-23,9)\end{array}$ \\
\hline Consumo regular de feijão & $\begin{array}{c}71,2 \\
(70,4-72,0)\end{array}$ & $\begin{array}{c}76,3 \\
(74,6-77,9)\end{array}$ & $\begin{array}{c}50,0 \\
(49,0-51,0)\end{array}$ & $\begin{array}{c}48,3 \\
(46,5-50,1)\end{array}$ & $\begin{array}{c}69,2 \\
(69,4-70,0)\end{array}$ & $\begin{array}{c}79,5 \\
(78,2-80,7)\end{array}$ \\
\hline Consumo regular de peixe & $\begin{array}{c}55,2 \\
(54,2-56,2)\end{array}$ & $\begin{array}{c}50,8 \\
(48,3-53,2)\end{array}$ & $\begin{array}{c}73,8 \\
(72,9-74,7)\end{array}$ & $\begin{array}{c}78,9 \\
(77,3-80,3)\end{array}$ & $\begin{array}{c}68,1 \\
(67,3-39,9)\end{array}$ & $\begin{array}{c}60,4 \\
(59,0-61,9)\end{array}$ \\
\hline \multicolumn{7}{|l|}{ Não saudáveis } \\
\hline $\begin{array}{l}\text { Consumo de carne ou frango } \\
\text { com excesso de gordura }\end{array}$ & $\begin{array}{c}35,8 \\
(34,9-36,7)\end{array}$ & $\begin{array}{c}45,8 \\
(43,9-47,8)\end{array}$ & $\begin{array}{c}31,7 \\
(30,7-32,6)\end{array}$ & $\begin{array}{c}42,3 \\
(40,5-44,1)\end{array}$ & $\begin{array}{c}26,2 \\
(25,5-26,9)\end{array}$ & $\begin{array}{c}33,1 \\
(31,7-37,5)\end{array}$ \\
\hline $\begin{array}{l}\text { Consumo regular de } \\
\text { refrigerantes }\end{array}$ & $\begin{array}{c}24,9 \\
(24,2-25,7)\end{array}$ & $\begin{array}{c}13,5 \\
(12,4-14,8)\end{array}$ & $\begin{array}{c}26,2 \\
(25,3-27,1)\end{array}$ & $\begin{array}{c}11,6 \\
(10,5-12,8)\end{array}$ & $\begin{array}{c}16,8 \\
(16,2-17,5)\end{array}$ & $\begin{array}{c}12,4 \\
(11,4-13,4)\end{array}$ \\
\hline $\begin{array}{l}\text { Consumo regular de } \\
\text { alimentos doces }\end{array}$ & $\begin{array}{c}22,0 \\
(21,3-22,7)\end{array}$ & $\begin{array}{c}19,5 \\
(17,9-21,3)\end{array}$ & $\begin{array}{c}12,2 \\
(11,6-12,9)\end{array}$ & $\begin{array}{c}9,0 \\
(8,0-10,1)\end{array}$ & $\begin{array}{c}18,3 \\
(17,7-19,0)\end{array}$ & $\begin{array}{c}15,0 \\
(14,0-16,1)\end{array}$ \\
\hline $\begin{array}{l}\text { Substituição de refeições por } \\
\text { lanches }\end{array}$ & $\begin{array}{c}4,3 \\
(3,9-4,7) \\
\end{array}$ & $\begin{array}{c}2,1 \\
(1,6-2,9) \\
\end{array}$ & $\begin{array}{c}4,1 \\
(3,7-4,5) \\
\end{array}$ & $\begin{array}{c}1,2 \\
(0,9-1,7)\end{array}$ & $\begin{array}{c}3,5 \\
(3,2-3,9) \\
\end{array}$ & $\begin{array}{c}1,8 \\
(1,4-2,2) \\
\end{array}$ \\
\hline \multirow{3}{*}{ Marcadores da alimentação } & \multicolumn{6}{|c|}{ Prevalência \% (IC95\%) } \\
\hline & \multicolumn{2}{|c|}{ Sudeste } & \multicolumn{2}{|c|}{ Sul } & \multicolumn{2}{|c|}{ Centro-Oeste } \\
\hline & Urbano & Rural & Urbano & Rural & Urbano & Rural \\
\hline \multicolumn{7}{|l|}{ Saudáveis } \\
\hline $\begin{array}{l}\text { Consumo recomendado de } \\
\text { FH }\end{array}$ & $\begin{array}{c}44,1 \\
(43,2-45,0)\end{array}$ & $\begin{array}{c}40,3 \\
(37,9-42,7)\end{array}$ & $\begin{array}{c}36,2 \\
(35,1-37-4)\end{array}$ & $\begin{array}{c}36,2 \\
(33,3-39,1)\end{array}$ & $\begin{array}{c}46,9 \\
(45,7-48,1)\end{array}$ & $\begin{array}{c}44,0 \\
(41,0-47,1)\end{array}$ \\
\hline Consumo regular de feijão & $\begin{array}{c}75,7 \\
(74,9-76,4)\end{array}$ & $\begin{array}{c}88,4 \\
(87,0-90,0)\end{array}$ & $\begin{array}{c}55,8 \\
(54,6-57,6)\end{array}$ & $\begin{array}{c}65,5 \\
(62,6-68,3)\end{array}$ & $\begin{array}{c}77,4 \\
(76,3-78,4)\end{array}$ & $\begin{array}{c}85,2 \\
(82,9-87,2)\end{array}$ \\
\hline Consumo regular de peixe & $\begin{array}{c}54,5 \\
(53,7-55,4)\end{array}$ & $\begin{array}{c}42,3 \\
(40,0-44,8)\end{array}$ & $\begin{array}{c}48,8 \\
(47,6-50,0)\end{array}$ & $\begin{array}{c}35,9 \\
(33,0-38,8)\end{array}$ & $\begin{array}{c}45,5 \\
(44,3-46,8)\end{array}$ & $\begin{array}{c}43,5 \\
(40,6-46,6)\end{array}$ \\
\hline \multicolumn{7}{|l|}{ Não saudáveis } \\
\hline $\begin{array}{l}\text { Consumo de carne ou frango } \\
\text { com excesso de gordura }\end{array}$ & $\begin{array}{c}34,1 \\
(33,3-34,9)\end{array}$ & $\begin{array}{c}55,3 \\
(52,9-57,8)\end{array}$ & $\begin{array}{c}35,6 \\
(34,4-36,8)\end{array}$ & $\begin{array}{c}54,2 \\
(51,2-57,2)\end{array}$ & $\begin{array}{c}42,7 \\
(41,5-43,9)\end{array}$ & $\begin{array}{c}57,2 \\
(54,2-60,2)\end{array}$ \\
\hline $\begin{array}{l}\text { Consumo regular de } \\
\text { refrigerantes }\end{array}$ & $\begin{array}{c}24,5 \\
(23,8-25,3)\end{array}$ & $\begin{array}{c}22,4 \\
(20,5-24,6)\end{array}$ & $\begin{array}{c}27,2 \\
(26,1-28,3)\end{array}$ & $\begin{array}{c}16,2 \\
(14.1-18,6)\end{array}$ & $\begin{array}{c}27,0 \\
(25,9-28,1)\end{array}$ & $\begin{array}{c}18,8 \\
(16,6-21,3)\end{array}$ \\
\hline $\begin{array}{l}\text { Consumo regular de } \\
\text { alimentos doces }\end{array}$ & $\begin{array}{c}21,9 \\
(21,1-22,6)\end{array}$ & $\begin{array}{c}24,4 \\
(22,4-26,6)\end{array}$ & $\begin{array}{c}25,2 \\
(24,2-26,3)\end{array}$ & $\begin{array}{c}22,5 \\
(20,0-25,1)\end{array}$ & $\begin{array}{c}21,5 \\
(19,6-21,5)\end{array}$ & $\begin{array}{c}21,8 \\
(19,4-24,4)\end{array}$ \\
\hline $\begin{array}{l}\text { Substituição de refeições por } \\
\text { lanches }\end{array}$ & $\begin{array}{c}8,5 \\
(8,0-9,0)\end{array}$ & $\begin{array}{c}5,4 \\
(4,4-6,7)\end{array}$ & $\begin{array}{c}10,5 \\
(9,8-11,3)\end{array}$ & $\begin{array}{c}6,3 \\
(5,0-8,0)\end{array}$ & $\begin{array}{c}7,9 \\
(7,3-8,6)\end{array}$ & $\begin{array}{c}3,2 \\
(2,2-4,4)\end{array}$ \\
\hline
\end{tabular}

Nota: $\mathrm{FH}=$ frutas e hortaliças.

Fonte: Elaborado pelas autoras, 2019.

preparo demanda maior tempo e habilidades culinárias, resultando em perda significativa para a cultura alimentar tradicional do país ${ }^{19}$.

Um alimento considerado como tradicional da cultura alimentar do brasileiro que vive em áreas rurais - as carnes com excesso de gordura - também foi mais prevalente nestas localidades. Estes resultados se assemelham àqueles obtidos em estudo realizado com amostra de adultos residentes nas áreas rurais de um estado da macrorregião Sudeste do Brasil que identificou pre- valência superior de $70 \%$ de consumo de gordura animal $^{20}$. Mas, apesar de alimento tradicional, as carnes gordurosas são consideradas como marcador da alimentação não saudável por seu consumo excessivo se associar ao risco de desenvolvimento de doenças cardiovasculares ${ }^{21}$. O maior consumo destes alimentos no Centro-Oeste e Sul pode se relacionar a aspectos culturais e econômicos, uma vez que estas macrorregiões se destacam pela atividade agropecuária ${ }^{22}$, favorecendo o acesso (preço e disponibilidade), além de seu 
Tabela 3. Valores de odds ratio para marcadores da alimentação saudável e não saudável segundo a residência em área urbana ou rural de população adulta brasileira. Pesquisa Nacional de Saúde, Brasil, 2013.

\begin{tabular}{|c|c|c|c|c|}
\hline Marcadores da alimentação & $\begin{array}{c}\text { OR não } \\
\text { ajustado }\end{array}$ & $\begin{array}{c}\mathrm{OR}^{\star} \\
\text { ajustado }^{\mathrm{a}}\end{array}$ & $\begin{array}{c}\mathrm{OR}^{\star} \\
\text { ajustado }^{\mathrm{b}}\end{array}$ & $\begin{array}{c}\mathrm{OR}^{\star} \\
\text { ajustadoc }^{\mathrm{c}}\end{array}$ \\
\hline \multicolumn{5}{|l|}{ Saudáveis } \\
\hline Consumo recomendado de frutas e hortaliças & $\begin{array}{c}0,73 \\
(0,70-0,76)\end{array}$ & $\begin{array}{c}0,73 \\
(0,70-0,77)\end{array}$ & $\begin{array}{c}0,83 \\
(0,80-0,88)\end{array}$ & $\begin{array}{c}0,89 \\
(0,85-0,96)\end{array}$ \\
\hline Consumo regular de feijão & $\begin{array}{c}1,26 \\
(1,21-1,33)\end{array}$ & $\begin{array}{c}1,23 \\
(1,18-1,29)\end{array}$ & $\begin{array}{c}1,07 \\
(1,02-1,13)\end{array}$ & $\begin{array}{c}1,20 \\
(1,14-1,26)\end{array}$ \\
\hline Consumo regular de peixe & $\begin{array}{c}0,94 \\
(0,90-0,98)\end{array}$ & $\begin{array}{c}0,93 \\
(0,89-0,97)\end{array}$ & $\begin{array}{c}1,00 \\
(0,96-1,05)\end{array}$ & $\begin{array}{c}0,88 \\
(0,84-0,92)\end{array}$ \\
\hline \multicolumn{5}{|l|}{ Não saudáveis } \\
\hline Consumo de carne ou frango com excesso de gordura & $\begin{array}{c}1,57 \\
(1,50-1,64)\end{array}$ & $\begin{array}{c}1,51 \\
(1,45-1,59)\end{array}$ & $\begin{array}{c}1,37 \\
(1,30-1,43)\end{array}$ & $\begin{array}{c}1,48 \\
(1,42-1,55)\end{array}$ \\
\hline Consumo regular de refrigerantes & $\begin{array}{c}0,56 \\
(0,53-0,60)\end{array}$ & $\begin{array}{c}0,55 \\
(0,52-0,59)\end{array}$ & $\begin{array}{c}0,52 \\
(0,49-0,55)\end{array}$ & $\begin{array}{c}0,55 \\
(0,52-0,59)\end{array}$ \\
\hline Consumo regular de alimentos doces & $\begin{array}{c}0,80 \\
(0,76-0,85)\end{array}$ & $\begin{array}{c}0,81 \\
(0,77-0,86)\end{array}$ & $\begin{array}{c}0,93 \\
(0,88-0,99)\end{array}$ & $\begin{array}{c}1,00 \\
(0,93-1,05)\end{array}$ \\
\hline Substituição de refeições por lanches & $\begin{array}{c}0,41 \\
(0,36-0,46)\end{array}$ & $\begin{array}{c}0,41 \\
(0,37-0,47)\end{array}$ & $\begin{array}{c}0,52 \\
(0,46-0,59)\end{array}$ & $\begin{array}{c}0,59 \\
(0,51-0,66)\end{array}$ \\
\hline
\end{tabular}

Nota: em negrito: associações estatisticamente significativas. ${ }^{\star}$ Urbano categoria de referência. ajustado por sexo e idade; ${ }^{\text {bajustado }}$ por sexo, idade, cor da pele e escolaridade; cajustado por sexo, idade, cor da pele, escolaridade e macrorregião do país.

Fonte: Elaborado pelas autoras, 2019.

forte valor simbólico representado pela cultura do churrasco nestas regiões ${ }^{23}$.

No que se refere ao consumo de alimentos ultraprocessados, o menor consumo de refrigerante verificado nas áreas rurais pode revelar possivelmente uma "proteção" dos hábitos alimentares tradicionais nestas áreas, o que precisa ser mantido. Entretanto, esforços precisam ser empreendidos para conter o avanço do consumo das bebidas açucaradas, haja vista a importante associação entre o consumo destas bebidas e o excesso de peso e as $\mathrm{DCNT}^{24}$. Para isto, a adoção de macropolíticas, como a taxação e mudanças nas rotulagens dos alimentos como experimentado em outros países, é essencial ${ }^{25-27}$.

Mas, apesar desse padrão alimentar tradicional baseado principalmente em alimentos minimamente processados, as áreas rurais também apresentaram menores prevalências de consumo de alimentos in natura e minimamente processados, como frutas e hortaliças, e peixes, respectivamente.

As diferenças no consumo de peixes entre as áreas rurais e urbanas podem derivar do custo elevado e menor disponibilidade deste alimento nas áreas rurais ${ }^{28}$, em especial aquelas em que a pesca não é atividade tradicional de subsistência. Ademais, é preciso reconhecer diferenças no território, como, por exemplo, na macrorregião
Norte que apresentou prevalências de consumo superior a $70 \%$ tanto nas áreas urbanas quanto rurais, evidenciando possivelmente diferenças da cultura alimentar. De toda forma estes resultados revelam a necessidade de estimular o consumo deste alimento no país, haja vista seu valor nutricional e facilidade de acesso em áreas litorâneas, e o potencial para expansão da pesca no país como forma de garantir a segurança alimentar $\mathrm{e}$ nutricional ${ }^{29}$.

O consumo de frutas e hortaliças é ainda insuficiente em todo país. Entretanto, quase o dobro de indivíduos residentes nas áreas urbanas relatou consumo recomendado, em comparação aos das áreas ruraii ${ }^{30}$. Tais diferenças podem indicar distinções em relação à disponibilidade destes alimentos e preços praticados ${ }^{31}$. Estudo realizado no Canadá, por exemplo, verificou menor acesso de FH nas áreas rurais, com impactos negativos sobre o consumo ${ }^{32,33}$. Outro estudo realizado em 18 países, incluindo o Brasil, mostrou que é necessário um dispêndio financeiro maior nas áreas rurais em comparação com as urbanas para consumir a quantidade recomendada desses alimen$\operatorname{tos}^{33}$.

O incentivo à agricultura familiar, bem como a prática de preços acessíveis, economia solidária e do cooperativismo rural são estratégias com potencial para aumentar o consumo de $\mathrm{FH}$ 
e beneficiar a saúde desta população, uma vez que boa parte da produção brasileira se destina à exportação e não são consumidas por quem produz. Estudo qualitativo que investigou as questões subjetivas relacionadas ao consumo de FH entre agricultores da zona rural de São Paulo mostrou que, para os fruticultores a fruta não é comida, e sim trabalho, portanto, não possuem função de alimentar, seu consumo não é essencial, e sua produção tem como função garantir o sustento familiar. Dessa forma, apesar de cultivadas e consumidas rotineiramente, as frutas são consideradas alimento somente quando compradas. As hortaliças, por sua vez, foram classificadas como alimentos de importância secundária ${ }^{34}$. Além disso, diante do delineamento seccional da PNS não foi possível mensurar a influência da sazonalidade no consumo de $\mathrm{FH}$, tanto nas áreas rurais quanto nas urbanas. Dessa forma, sugerese a realização de estudo que considere possíveis padrões sazonais de consumo de acordo com diferentes períodos de coleta dos dados da PNS.

O consumo alimentar unifica o país do ponto de vista da nutrição e ao mesmo tempo revela sua história, cultura, tradições e como ele vivencia o processo de urbanização e de globalização ${ }^{23}$. A urbanização traz consigo uma série de mudanças no consumo alimentar advindas da falta de tempo para preparo e compra dos alimentos, o que pode repercutir na escolha por uma alimentação mais prática e com maior participação de alimentos ultraprocessados ${ }^{20,35,36}$. Isto pode revelar, de certa forma, uma provável penalização por se viver no meio urbano ${ }^{11}$ uma vez que, a busca de praticidade pode acarretar em maior participação de alimentos prontos para consumo na alimentação, de refeições realizadas fora do domicílio e substituição de refeições por lanches ${ }^{35,36}$. Como exemplo desta questão, neste estudo, citase as diferenças identificadas nas prevalências de substituição do almoço e jantar por sanduíches, salgados ou pizzas entre os brasileiros residentes nas áreas urbanas e rurais. Entre 1974 e 2003 havia uma prevalência até três vezes menor de consumo de refeições prontas e industrializadas nas áreas rurais em comparação às urbanas ${ }^{37}$. Da mesma forma, dados da Pesquisa de Orçamentos Familiares (2008-2009) mostraram menor prevalência da realização da alimentação fora do domicílio nas áreas rurais ${ }^{38}$.

Paralelamente à urbanização, a globalização gera uma tendência de redução das diferenças regionais devido à maior chance de integração, troca de informações e alcance de alimentos. Dessa forma, acredita-se que a tendência é que cada vez mais residentes das áreas rurais vão aderir aos padrões alimentares das áreas urbanas, logo, é de suma importância a forte atuação de políticas públicas para a promoção da alimentação adequada e saudável que valorizem a cultura alimentar tradicional dos brasileiros, incluindo medidas regulatórias que contribuam para a construção de ambientes alimentares saudáveis. Promover ambientes alimentares saudáveis e valorizar os já existentes são medidas cruciais para favorecer e fortalecer mudanças individuais e potencializar iniciativas da população. Ademais, nos espaços rurais requer o reconhecimento de suas potencialidades como ambientes promotores da saúde, com valorização de tradições e raízes culturais locais.

Este estudo apresenta limitações que devem ser consideradas. Inquéritos alimentares são passíveis de viés de informação, prejudicando a aferição da dieta habitual. Além disso, vale ressaltar que por ser um país de dimensão continental e grande diversidade, as áreas rurais e urbanas podem não ser homogêneas, e a estrutura e geografia múltiplas afetar os resultados. Entretanto, este estudo tem poder amostral para ser representativo destas áreas, considerando toda a extensão territorial brasileira.

Por outro lado, como destaque positivo do estudo aponta-se a realização de análise dos dados ajustadas por possíveis variáveis confundidoras, estratégia estatística até então não explorada em estudos anteriores que utilizaram a mesma base de dados. Por fim, é evidente a importância deste estudo ao trabalhar com a Pesquisa Nacional de Saúde, que é representativa da população adulta brasileira de todas as macrorregiões, sobretudo com uma perspectiva de compreender melhor as dinâmicas alimentares das áreas urbanas e rurais de um país com reconhecida diversidade como o Brasil, o que abre horizontes para intervenções nutricionais nestas regiões de acordo com suas peculiaridades.

Conclui-se que há diferenças no consumo alimentar dos brasileiros residentes em áreas urbanas e rurais. Pondera-se, entretanto, que com o avanço da urbanização e da industrialização há uma tendência de redução destas diferenças. Este estudo revela a importância de que as políticas de alimentação e nutrição, bem como as orientações para a prática de uma alimentação adequada e saudável ofertadas aos brasileiros sejam consonantes não somente com o contexto econômico e social vivenciado, mas que igualmente respeitem e valorizem as dimensões culturais da alimentação visando sua factibilidade, sustentabilidade e promoção de bem-estar à população. 


\section{Colaboradores}

DVP Costa, MS Lopes, RD Mendonça, PP Freitas e ACS Lopes participaram da concepção do projeto, análise e interpretação dos dados. DC Malta concebeu e coordenou a Pesquisa Nacional de Saúde; coordenou a metodologia e questionários utilizados, e o plano de análises. DVP Costa, MS Lopes e PP Freitas foram responsáveis pela redação do artigo. E todas as autoras foram responsáveis pela revisão final do artigo e sua aprovação.

\section{Referências}

1. Malta DC, Andrade SSCA, Stopa SR, Pereira CA, Szwarcwald CL, Silva Júnior JB, Reis AAR. Estilos de vida da população brasileira: resultados da Pesquisa Nacional de Saúde, 2013. Epidemiol Serv Saude 2015; 24(2):217-226

2. World Health Organization (WHO). Diet, nutrition and the prevention of chronic diseases: report of a Joint WHO/FAO Expert Consultation. Genebra: WHO; 2003.

3. Popkin BM. Urbanization, Lifestyle Changes and the Nutrition Transition. World Develop 1999; 27(11):1905-1916.

4. Brasil. Ministério da Saúde (MS). Departamento de Atenção Básica. Secretaria de Atenção à Saúde. Guia alimentar para a população brasileira. 2a ed. Brasília: MS; 2014.

5. Souza-Júnior PRB, Freitas MPS, Antonaci GA, Szwarcwald CL. Desenho da amostra da Pesquisa Nacional de Saúde 2013. Epidemiol Serv Saude 2015; 24(2):207216.

6. Martins APB, Levy RB, Claro RM, Moubarac JC, Monteiro CA. Participação crescente de produtos ultraprocessados na dieta brasileira (1987-2009). Rev Saude Publica 2013; 47(4):656-665.

7. Canella DS, Levy RB, Martins AP, Claro RM, Moubarac JC, Baraldi LG, Cannon G, Monteiro CA. Ultra-processed food products and obesity in Brazilian households (2008-2009). PLOS ONE 2014; 9(3):e92752.

8. Monteiro CA, Cannon G, Levy RB, Moubarac JC, Louzada ML, Rauber F, Khandpur N, Cediel D, Neri D, Martinez-Steele E, Baraldi LG, Jaime PC. Ultra -processed foods: what they are and how to identify them. Public Health Nutr 2019; 2:1-6.

9. United Nations (UN). World Urbanization Prospects, 2014 [Internet]. [acessado $2017 \mathrm{dez}$ 1]. Disponível em: https://esa.un.org/unpd/wup/publications/files/ wup2014-highlights.pdf.

10. Instituto Brasileiro de Geografia e Estatística (IBGE). Sinopse do Censo Demográfico 2010. Rio de Janeiro: IBGE; 2011.

11. Brasil. Ministério da Saúde (MS). Alimentos regionais brasileiros. 2a ed. Brasília: MS; 2015.

12. Caiaffa WT, Ferreira FR, Ferreira AD, Oliveira CL, Camargos VP, Proietti FA. Saúde urbana: "a cidade é uma estranha senhora, que hoje sorri e amanhã te devora". Cien Saude Colet 2008; 13(6):1785-1796.

13. Instituto Brasileiro de Geografia e Estatística (IBGE). Pesquisa Nacional de Saúde 2013: percepção do estado de saúde, estilos de vida e doenças crônicas. Rio de Janeiro: IBGE; 2014.

14. Mendes LL, Campos SF, Malta DC, Bernal RTI, Sá NNBD, Velásquez-Meléndez G. Validade e reprodutibilidade de marcadores do consumo de alimentos e bebidas de um inquérito telefônico realizado na cidade de Belo Horizonte (MG), Brasil. Rev Bras Epidemiol 2011; 14(Supl. 1):S80-S89.

15. Jaime PC, Stopa SR, Oliveira TP, Vieira ML, Szwarcwald CL, Malta DC. Prevalência e distribuição sociodemográfica de marcadores de alimentação saudável, Pesquisa Nacional de Saúde, Brasil 2013. Epidemiol Serv Saude 2015; 24(2):267-276. 
16. Souza AM, Pereira RA, Yokoo EM, Levy RB, Sichieri R. Alimentos mais consumidos no Brasil: Inquérito Nacional de Alimentação 2008-2009. Rev Saude Publica 2013; 47(Supl. 1):190s-199s.

17. Brasil. Ministério da Saúde (MS). Secretaria de Vigilância em Saúde. Secretaria de Gestão Estratégica e Participativa. Vigitel Brasil 2016: vigilância de fatores de risco e proteção para doenças crônicas por inquérito telefônico. Brasília: MS; 2017.

18. Panikolaou Y, Fulgoni VL 3rd. Bean consumption is associated with greater nutrient intake, reduced systolic blood pressure, lower body weight, and a smaller waist circumference in adults: results from the National Health and Nutrition Examination Survey 19992002. J Am Cool Nutr 2008; 27(5):569-576.

19. Schlindwein MM, Kassouf AL. Mudanças no padrão de consumo de alimentos tempo-intensivos e de alimentos poupadores de tempo, por região do Brasil. In: Gasto e consumo das famílias brasileiras contemporâneas. Brasília: IPEA; 2006-2007. p.423-462.

20. Carvalho EO, Rocha EF. Consumo alimentar de população adulta residente em área rural da cidade de Ibatiba (ES, Brasil). Cien Saude Colet 2011; 16(1):179185.

21. Kris-Etherton PM, Petersen K, Van Horn L. Convincing evidence supports reducing saturated fat to decrease cardiovascular disease risk. BMJ Nutr Prev Health 2018; 1:5-6.

22. Castro CN. A agropecuária na região Centro-Oeste: Limitações ao desenvolvimento e desafios futuros. Texto para Discussão, no 1923. Brasília: IPEA; 2014.

23. Canesqui AM, Garcia RWD. Antropologia e nutrição: um diálogo possível. Rio de Janeiro: Editora Fiocruz; 2005.

24. Malik VS, Pan N, Willett WC, Hu FB. Sugar-sweetened beverages and weight gain in children and adults: a systematic review and meta-analysis. Am J Clinical Nutr 2013; 98(4):1084-1102.

25. Organización Panamericana de la Salud (OPAS). Experiencia de México en el establecimiento de impuestos a las bebidas azucaradas como estrategia de salud pública. México: OPAS; 2015.

26. Veerman JL, Sacks G, Antonopoulos N, Martin J. The Impact of a Tax on Sugar-Sweetened Beverages on Health and Health Care Costs: A Modelling Study. PLoS ONE 2016; 11(4):e0151460.

27. Sánchez VS, Silva CV. Impacto de la nueva ley de etiquetados de alimentos en la venta de productos en Chile. Perfiles Econom 2017; 3:7-33.

28. Mazengo MC, Simell O, Lukmanji Z, Shirima R, Karvetti RL. Food consumption in rural and urban Tanzania. Acta Tropica 1997; 68:313-326.

29. Duran AC, Diez Roux Av, Latorre MR, Jaime PC. Neighborhood socioeconomic characteristics and differences in the availability of healthy food stores and restaurants in São Paulo, Brazil. Health Place 2013; 23:39-47.

30. Jaime PC, Monteiro CA. Fruit and vegetable intake by Brazilian adults, 2003. Cad Saude Publica 2005; 21(Supl. 1):19-24.
31. Jaime PC, Stopa SR, Oliveira TP, Vieira ML, Szwarcwald CL, Malta DC. Prevalência e distribuição sociodemográfica de marcadores de alimentação saudável, Pesquisa Nacional de Saúde, Brasil 2013. Epidemiol Serv Saude 2015; 24(2):267-276.

32. Pouliot N, Hamelin AM. Disparities in fruit and vegetable supply: A potential health concern in the greater Québec City area. Public Health Nutr 2009; 12(11):2051-2059.

33. Miller V, Yusuf S, Chow CK, Dehghan M, Corsi DJ, Lock K, Popkin B, Rangarajan S, Khatib R, Lear SA, Mony P, Kaur M, Mohan V, Vijayakumar K, Gupta R, Kruger A, Tsolekile L, Mohammadifard N, Rahman $\mathrm{O}$, Rosengren A, Avezum A, Orlandini A, Ismail N, Lopez-Jaramillo P, Yusufali A, Karsidag K, Iqbal R, Chifamba J, Oakley SM, Ariffi F, Zatonska K, Poirier P, Wei L, Jian B, Hui C, Xu L, Xiulin B, Teo K, Mente A. Availability, affordability, and consumption of fruits and vegetables in 18 countries across income levels: findings from the Prospective Urban Rural Epidemiology (PURE) study. Lancet Glob Health 2016; 4(10):e695-e703.

34. Alves HJ, Boog MCF. Representações sobre consumo de frutas, verduras e legumes entre fruticultores de zona rural. Rev Nutr 2008; 2(6):705-715.

35. Claro RM, Maia EG, Costa BVL, Diniz DP. Preço dos alimentos no Brasil: prefira preparações culinárias a alimentos ultraprocessados. Cad Saude Publica 2016; 32(8):e00104715.

36. Garcia RWD. Reflexos da globalização na cultura alimentar: considerações sobre as mudanças na alimentação urbana. Rev Nutr 2003; 16(4):483-492.

37. Levy-Costa RB, Sichieri R, Pontes NS, Monteiro CA Disponibilidade domiciliar de alimentos no Brasil: distribuição e evolução (1974-2003). Rev Saude Publica 2005; 39(4):530-540.

38. Bezerra IN, Souza AM, Pereira RA, Sichieiri R. Consumo de alimentos fora do domićlio no Brasil. Rev Saude Publica 2013; 47(Supl. 1):200s-211s.

Artigo apresentado em 10/09/2019

Aprovado em 04/12/2019

Versão final apresentada em 06/12/2019

Editores-chefes: Romeu Gomes, Antônio Augusto Moura da Silva 
